Research Article

\title{
Prognostic Evaluation for Patients over 45 Years Old with Gallbladder Adenocarcinoma Resection: A SEER-Based Nomogram Analysis
}

\author{
Pengfei Li $\mathbb{D}$ and Lujun Song $\mathbb{D}$ \\ Department of General Surgery, Zhongshan Hospital, Fudan University, Shanghai 200032, China \\ Correspondence should be addressed to Lujun Song; yylslj@hotmail.com
}

Received 8 April 2020; Revised 16 June 2020; Accepted 29 June 2020; Published 18 July 2020

Academic Editor: Giuseppe Piccinni

Copyright (C) 2020 Pengfei Li and Lujun Song. This is an open access article distributed under the Creative Commons Attribution License, which permits unrestricted use, distribution, and reproduction in any medium, provided the original work is properly cited.

\begin{abstract}
Gallbladder adenocarcinoma is the main histopathological type of gallbladder cancer (GBC), so it is particularly important to understand its biological characteristics. Due to the low incidence of this type of cancer, there are few studies with large sample sizes. The log of positive lymph nodes (LODDS) has been evaluated by many scholars as a lymph node stage that may play a better role than the $8^{\text {th }}$ edition of the American Joint Committee on Cancer (AJCC) lymph node staging system in many cancers. However, the effect of LODDS has not been proven in gallbladder adenocarcinoma. Our research aimed to identify independent prognostic factors that are closely related to overall survival (OS) in patients with gallbladder adenocarcinoma over 45 years of age using data from the Surveillance, Epidemiology and, End Results (SEER) database. All patients were randomly divided into a modeling cohort and an internal validation cohort. Seven independent prognostic factors associated with OS - age, marital status, grade, tumor size, AJCC $8^{\text {th }}$ edition T stage and M stage, and LODDS—-were used to build a nomogram to predict 1-, 3-, and 5-year survival. The C-index of our nomogram was 0.735 (95\% CI, 0.716 to 0.754 ), and together with the calibration curve and ROC curve validation, the results confirmed the prediction effect of our nomogram. We believe that our nomogram will be an accurate and convenient method for patient prognosis assessment in the future.
\end{abstract}

\section{Introduction}

Gallbladder cancer (GBC) is the most common tumor of the biliary tract with the worst overall survival [1]. This is because GBC is usually diagnosed when the tumor has progressed and is already large enough to block nearby structures [2]; therefore, the overall survival (OS) rate of GBC is still low: the 5-year OS rate of unresectable GBC is no more than 5\% [3-5]. At the very beginning, most patients only have gallstones in their gallbladder [2]. If not handled promptly, chronic inflammation caused by gallstones can stimulate the gallbladder mucosa and eventually lead to GBC [2, 5]. However, radical surgery can significantly improve patient prognosis regardless of the tumor stage. Therefore, the early identification and management of patients with a good prognosis is very important.
Gallbladder adenocarcinoma is the main type of GBC, accounting for approximately $80 \%[6,7]$. According to data from the Surveillance, Epidemiology, and End Results (SEER) database, $97.3 \%$ of patients with GBC are middleaged (older than 45), so the evaluation of good prognosis in this patient group is urgent. Although many studies have reported some prognostic factors for GBC, including age, TNM stage, tumor size, adjuvant therapy, and pathological grade, there has not yet been a population-based study that has systematically clarified postoperative patients over 45 years of age $[6,8,9]$. The $8^{\text {th }}$ edition of the AJCC TNM staging system has just been put into clinical use, so it is urgent to establish a prediction model based on this staging system to assist in daily clinical use [10]. The log of positive lymph nodes (LODDS) has been evaluated by many scholars as a lymph node stage that may play a better role than the $8^{\text {th }}$ 
edition of the AJCC lymph node staging system [11, 12]. A better prognosis prediction model could be established by combining these two different evaluation models. The aim of our study is to use the SEER database to assess the independent prognostic factors for patients who underwent gallbladder adenocarcinoma resection and were older than 45 years old and to provide individualized guidance for the surgical prognosis of this population.

\section{Materials and Methods}

2.1. Patient Selection. We extracted the clinical data of patients with GBC from 2004 to 2016 from the SEER database, which covers up $28 \%$ of the US population and $97 \%$ of tumor categories. The exclusion criteria we used were as follows: (1) patients whose pathological type was not gallbladder adenocarcinoma or who did not have a pathological diagnosis; (2) patients whose age at diagnosis was younger than 45 years old; (3) patients whose surgery information was unclear or patients without surgery; and (4) patients with unclear data regarding sex, race, marital status, TNM stage, pathological grade, tumor size, lymph node surgery scope, radiation, chemotherapy, or regional node examination information. Our data selection method is shown in Figure 1 and Table S1. The selected data were divided into a training cohort and a validation cohort by a ratio of approximately 3 to 1 , the same as in previous similar studies $[13,14]$. Data downloaded from the SEER database did not require patients' informed consent and may be reproduced or copied without permission.

In our research, we used the variables of sex, race, marital status, age at diagnosis, AJCC TNM stage, pathological grade, lymph node surgery scope, tumor size, radiation, and chemotherapy. The $8^{\mathrm{th}}$ AJCC TNM staging system was used to identify the stage of gallbladder adenocarcinoma [10]. The variable RX Sum (1998+) was used to extract tumor size. The tumor's histologic subtype was identified by specific coding data embedded in the SEER database; i.e., code 8140 represented adenocarcinoma. LODDS was calculated by using the following formula: $\log [(0.5+$ the amounts of positive LNs $) /(0.5+$ the amounts of negative LNs $)]$ [11]. The LODDS value in our cohort ranged from -4.39 to 3.30 . We used X-tile software to obtain the best cut-off values for LODDS and tumor size (Figure S1, 15). LODDS was divided into LODDS1 (-4.39 to -1.47$)$, LODDS2 ( -1.47 to $0.90)$, and LODDS3 (0.90 to 3.30$)$, and tumor size was grouped into $<1.7 \mathrm{~cm}, 1.7-3.3 \mathrm{~cm}$, and $\geq 3.4 \mathrm{~cm}$.

2.2. Statistical Analysis. SPSS 20.0 statistical software (SPSS Inc., Chicago, IL, USA) and R software version 3.6.1 (The R Foundation for Statistical Computing, Vienna, Austria. http://www.r-project.org) were used to run statistical analysis. The Pearson chi-squared test was used to describe the clinical characteristics. Univariate and multivariate analyses were conducted by the Cox proportional hazards regresslion model, and hazard ratios (HRs) together with the corresponding 95\% confidence intervals (CIs) were calculated. Variables with $P<0.05$ in univariate analysis were incorporated into multivariate analysis. The Kaplan-Meier method was used to estimate OS, and different survival curves were analyzed by log-rank tests. $P$ values were calculated by two sides, and $P<0.05$ was considered statistically significant for all of our analyses.

The rms package from $\mathrm{R}$ was used to formulate a nomogram based on the independent prognostic factors from multivariate analysis. The concordance index (C-index), Akaike information criterion (AIC), ROC curve, and calibration plots were used to evaluate the nomogram as described previously [14]. The C-index denoted a low accuracy between 0.50 and 0.70 , a medium accuracy between 0.71 and 0.90 , and a high accuracy over 0.90 . The calibration curve was a scatter plot of the actual and predicted incidence values in order to compare the predicted OS and the observed OS.

\section{Results}

3.1. Clinicopathologic Features of Patients. This present study identified 1612 patients with gallbladder adenocarcinoma between 2004 and 2016; 1212 patients were in the training cohort, and 400 patients were in the validation cohort. The clinicopathologic features of the patients in the training cohort and validation cohort are presented in Table 1 . There was no statistically significant difference between the two groups for all data. The majority of patients in both cohorts were relatively old ( $\geq 65$ years old), female, white and married. The most common tumor grade was moderately differentiated $(775,48.1 \%)$, followed by poorly differentiated (596, 37.0\%). According to the $8^{\text {th }}$ AJCC TNM staging system, the primary T-stage was $\mathrm{T} 2(802,49.8 \%)$, and the primary M-stage was M0 $(1365,84.7 \%)$. The majority of patients who underwent removal of 1-3 regional lymph nodes accounted for $67.0 \%$. Only 678 (42.1\%) patients underwent chemotherapy, and 385 (23.9\%) patients underwent radiation. There were 895 (55.5\%) patients with a tumor size of $1.9-4.8 \mathrm{~cm}, 355(22.0 \%)$ patients with a tumor size of $<1.9 \mathrm{~cm}$, and $362(22.5 \%)$ patients with a tumor size of $\geq 4.8 \mathrm{~cm}$. LODDS was divided into LODDS1 $(-4.39$ to -1.47$)$, LODDS2 (-1.47 to 0.90$)$, and LODDS3 (0.90 to 3.30) by X-tile software. The medium-risk cohort (LODDS2) consisted of $642(39.8 \%)$ patients, followed by LODDS1 $(518,32.1 \%)$ and LODDS2 (452, 28.0\%).

3.2. Prognostic OS Analysis by Univariate and Multivariate Cox Analyses. In general, the median follow-up time was 15 months (range, 0-154 months), and the median OS was 23 months (95\% CI: 20.52-25.47 months). The 1-, 3-, and 5 -year OS rates for all patients were $68.3 \%, 37.3 \%$, and $28.3 \%$, respectively. Through univariate testing for the training cohort, factors including age, marital status, race, grade, tumor size, $\mathrm{T}$ stage, LODDS, and $\mathrm{M}$ stage showed statistical significance with $\mathrm{OS}(P<0.05)$. In addition, sex, LN surgery scope, radiation, and chemotherapy failed to reach statistical significance $(P>0.05)$ (Table 2$)$.

Seven independent survival predictors associated with OS, age, marital status, grade, $\mathrm{T}$ stage, $\mathrm{M}$ stage, tumor size, and LODDS were selected by multivariate Cox regression analysis (Table 2). A patient who was unmarried ( $\mathrm{HR}=1.298,95 \%$ CI: $1.117-1.508, \quad P<0.001)$ and older than 65 years 


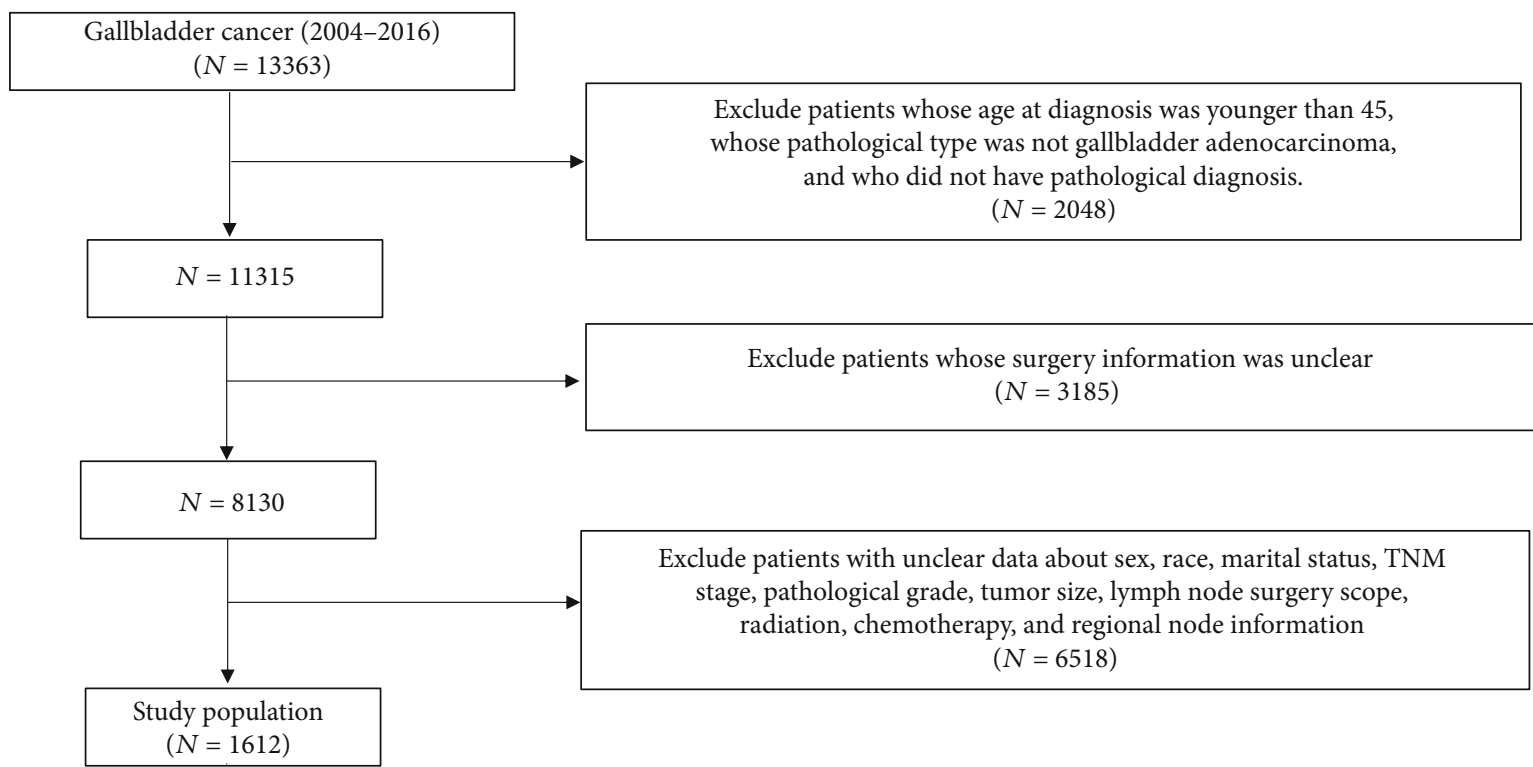

FIgURE 1: The study flow diagram of the selection process.

$(\mathrm{HR}=1.686,95 \% \mathrm{CI}: 1.4430-1.988, P<0.001)$ and who had poorly differentiated pathological grade IV $(\mathrm{HR}=2.295,95 \%$ CI: $0.898-5.066, P=0.005)$, T4 stage $(\mathrm{HR}=3.715,95 \% \mathrm{CI}$ : 2.262-6.101, $P<0.001)$, and M1 stage ( $\mathrm{HR}=2.067,95 \% \mathrm{CI}$ : 1.704-2.508, $P<0.001)$ disease as well as a tumor size larger than $4.8 \mathrm{~cm}(\mathrm{HR}=1.503,95 \% \mathrm{CI}: 1.179-1.916, P<0.001)$ was considered to have a worse prognosis. In particular, patients with LODDS2 (HR $=1.316,95 \% \mathrm{CI}: 1.087-1.593, P=0.005)$ or LODDS3 ( $\mathrm{HR}=2.515,95 \% \mathrm{CI}: 2.053-3.080, P<0.001$ ) appeared to have a worse prognosis than those with LODDS1. Through Kaplan-Meier survival analysis, we concluded that the above independent factors of prognosis had statistical significance for OS in their respective strata (Figure 2).

3.3. Prognostic Nomograms for OS. The prognostic nomogram was constructed by the independent survival predictors obtained from the results of the multivariate analysis described above (Figure 3). The $\mathrm{C}$-index for OS prediction in the training cohort and validation cohort was 0.735 (95\% CI, 0.716 to 0.754 ) and 0.740 (95\% CI, 0.721 to 0.759$)$. The C-index of the $8^{\text {th }}$ edition of the AJCC TNM staging system in the training cohort and validation cohort was 0.698 (95\% CI: $0.678-0.717$ ) and 0.693 (95\% CI: 0.674-0.712). The AIC of our nomogram in the training cohort and validation cohort was 8658 and 2324 . The AIC of the $8^{\text {th }}$ edition of the AJCC TNM staging system in the training cohort and validation cohort was 8763 and 2375. Our nomogram shows a better discrimination and prediction ability than the $8^{\text {th }}$ edition of the AJCC TNM staging system. The calibration curve and ROC curve for the survival rate in the training cohort and validation cohort showed good consistency between prediction and observation at one, three, and five years (Figures 4 and 5.

\section{Discussion}

In our research, we used the data from the SEER database to set up a nomogram for OS in approximately 1612 patients over 45 years old who underwent gallbladder adenocarcinoma resection. Although many established nomograms of GBC consider radiotherapy, chemoradiotherapy, and adenocarcinoma using the SEER database $[6,9,15,16]$, there is no GBC population-based research regarding specific pathology types or age groups based on LODDS.

Our study is the first population-based study using the SEER database to establish a prognostic nomogram for patients older than 45 who underwent gallbladder adenocarcinoma resection based on the $8^{\text {th }}$ edition of the AJCC TNM staging system and LODDS. In our study, seven independent prognostic factors, age, marital status, grade, tumor size, AJCC $8^{\text {th }}$ edition T stage, AJCC $8^{\text {th }}$ edition $M$ stage, and LODDS, were selected. Based on these factors, we built a nomogram to evaluate the survival rate for the patients described above.

Through multivariate Cox regression analysis, we found that patients older than sixty-five years may have a relatively poor overall survival. Our results are consistent with those of previous studies showing that elderly patients tend to have higher tumor grades, worse performance statuses, and shorter survival times than younger patients $[17,18]$. Similar to the research of Song et al. [19], our study found that marital status actually affected the prognosis of patients with gallbladder adenocarcinoma. Patients who were unmarried or divorced would have relatively poor survival after surgery. Some studies have shown that unmarried patients are more likely to be affected by depression and anxiety from a biological and social psychological point of view because they have no spouse, which in turn affects their willingness to undergo follow-up treatment and their confidence in recovery after receiving treatment [19-21]. As a result, these patients have a worse prognosis than married patients. This theory was confirmed by our research. Tumor grade is closely related to the biological behavior of different kinds of tumors, so it naturally affects the prognosis of patients [1, 22]. In our research, poorly differentiated patients accounted for $37.0 \%$. 
TABLE 1: Clinical and pathological features of patients with gallbladder adenocarcinoma over 45 years old.

\begin{tabular}{|c|c|c|c|c|c|}
\hline Variable & Variable level & $\begin{array}{c}N \\
(n=1612) \\
n(\%)\end{array}$ & $\begin{array}{l}\text { Training cohort } \\
\qquad \begin{array}{c}n=1212) \\
n(\%)\end{array}\end{array}$ & $\begin{array}{l}\text { Validation cohort } \\
(n=400) \\
n(\%)\end{array}$ & $P$ value \\
\hline \multirow{2}{*}{ Age } & $<65$ & $543(33.7)$ & $412(34.0)$ & $131(32.8)$ & 0.648 \\
\hline & $\geq 65$ & $1069(66.3)$ & $800(66.0)$ & $269(67.2)$ & \\
\hline \multirow{2}{*}{ Marital status } & Married & $895(55.5)$ & $675(55.7)$ & $220(55.0)$ & 0.809 \\
\hline & Unmarried & $717(44.5)$ & $537(44.3)$ & $180(45.0)$ & \\
\hline \multirow{3}{*}{ Race } & White & $1213(75.2)$ & $917(75.7)$ & $296(74.0)$ & 0.362 \\
\hline & Black & $194(12.0)$ & $138(11.4)$ & $56(14.0)$ & \\
\hline & Other & $205(12.7)$ & $157(13.0)$ & $48(12.0)$ & \\
\hline \multirow{2}{*}{ Sex } & Male & $492(30.5)$ & $381(31.4)$ & $111(27.8)$ & 0.165 \\
\hline & Female & $1120(69.5)$ & $831(68.6)$ & $289(72.2)$ & \\
\hline \multirow{4}{*}{ Grade } & Well, I & $218(13.5)$ & $166(13.7)$ & $52(13.0)$ & 0.692 \\
\hline & Moderately, II & $775(48.1)$ & $580(47.9)$ & $195(48.8)$ & \\
\hline & Poorly, III & $596(37.0)$ & $451(37.2)$ & $145(36.2)$ & \\
\hline & Undifferentiated, IV & $23(1.4)$ & $15(1.2)$ & $8(2.0)$ & \\
\hline \multirow{4}{*}{ AJCC $8^{\text {th }} \mathrm{T}$ stage } & $\mathrm{T} 1$ & $150(9.3)$ & $112(9.2)$ & $38(9.5)$ & 0.363 \\
\hline & $\mathrm{T} 2$ & $802(49.8)$ & $604(49.8)$ & $198(49.5)$ & \\
\hline & $\mathrm{T} 3$ & $602(37.3)$ & $458(37.8)$ & $144(36)$ & \\
\hline & $\mathrm{T} 4$ & $58(3.6)$ & $38(3.14)$ & $20(5.0)$ & \\
\hline \multirow{3}{*}{ LODDS } & LODDS1 & $518(32.1)$ & $397(32.8)$ & $121(30.2)$ & 0.648 \\
\hline & LODDS2 & $642(39.8)$ & $478(39.4)$ & $164(41.0)$ & \\
\hline & LODDS3 & $452(28.0)$ & $337(27.8)$ & $115(28.7)$ & \\
\hline \multirow{2}{*}{ AJCC $8^{\text {th }} M$ stage } & M0 & $1365(84.7)$ & $1024(84.5)$ & $343(85.8)$ & 0.542 \\
\hline & M1 & $245(15.2)$ & $188(15.5)$ & $57(14.2)$ & \\
\hline \multirow{3}{*}{ LN surgery scope } & None & $40(2.5)$ & $31(2.6)$ & $9(2.3)$ & 0.942 \\
\hline & $1 \sim 3$ & $1080(67.0)$ & $811(66.9)$ & $269(67.2)$ & \\
\hline & $4 \sim$ & $492(30.5)$ & $370(30.5)$ & $122(30.5)$ & \\
\hline \multirow{2}{*}{ Radiation } & Yes & $385(23.9)$ & $299(24.7)$ & $86(21.5)$ & 0.197 \\
\hline & No/unknown & $1227(76.1)$ & $913(75.3)$ & $314(78.5)$ & \\
\hline \multirow{2}{*}{ Chemotherapy } & Yes & $678(42.1)$ & $513(42.3)$ & $165(41.2)$ & 0.705 \\
\hline & No/unknown & $934(57.9)$ & $699(57.7)$ & $235(58.8)$ & \\
\hline \multirow{3}{*}{ Tumor size $(\mathrm{cm})$} & $<1.9$ & $355(22.0)$ & $273(22.5)$ & $82(20.5)$ & 0.218 \\
\hline & $1.9 \sim 4.8$ & $895(55.5)$ & $657(54.2)$ & $238(59.5)$ & \\
\hline & $\geq 4.8$ & $362(22.5)$ & $282(23.3)$ & $80(20.0)$ & \\
\hline
\end{tabular}

AJCC: American Joint Committee on Cancer; LODDS: log of positive lymph nodes; LN: lymph nodes.

Our analysis and survival curve showed that when the tumor's pathological grade changed from better to worse, the patient's OS changed subsequently. This result is consistent with the conclusions from previous studies [1, 22]. The AJCC TNM stage is an important indicator for judging the degree of tumor progression, choosing treatment options, and determining prognosis $[10,23]$. In our results, the AJCC T stage and AJCC $M$ stage were considered to be independent prognostic factors. Lymph node metastasis is the most important prognostic factor, and many studies show that only adequate lymph node dissection will give patients a better prognosis [24-27]. In our research, we did not use traditional AJCC lymph node staging. Instead, we used a completely new method of lymph node staging: LODDS [27-29]. Unlike traditional lymph node staging, LODDS is a new method of lymph node staging that has been proposed for a long time. It has been well validated in many tumor models and has been shown to play a better role than the latest eighth version of lymph node staging for $\mathrm{GBC}[30,31]$. Compared with simply classifying lymph nodes based on the presence of lymph node metastasis or the number of lymph node metastases, LODDS can better eliminate the impact of the total number of lymph nodes removed by surgery on the detection rate of positive lymph nodes $[28,29]$. From our results, we can see that the degree of lymph node dissection has no practical value in judging the prognosis of patients, and similar results have been obtained in studies on pancreatic cancer [14]. This may be because only the number of total lymph nodes dissected was considered. Many studies have shown that the rate of positive lymph 
TABLE 2: Univariate and multivariate Cox regression analyses of OS for patients with gallbladder adenocarcinoma in the training cohort.

\begin{tabular}{|c|c|c|c|c|c|c|c|}
\hline \multirow{2}{*}{ Characteristics } & \multirow{2}{*}{ Variable level } & \multicolumn{3}{|c|}{ Univariate analysis } & \multicolumn{3}{|c|}{ Multivariate analysis } \\
\hline & & HR & $95 \% \mathrm{CI}$ & $P$ value & HR & $95 \% \mathrm{CI}$ & $P$ value \\
\hline \multirow{3}{*}{ Age (years) } & & & & $<0.001^{* * *}$ & & & $<0.001^{* * *}$ \\
\hline & $\leq 65$ & Reference & & & Reference & & \\
\hline & $>65$ & 1.55 & $1.317-1.824$ & $<0.001^{* * *}$ & 1.686 & $1.400-1.988$ & $<0.001^{* * *}$ \\
\hline \multirow{3}{*}{ Marital status } & & & & $0.016^{*}$ & & & $0.001^{* *}$ \\
\hline & Married & Reference & & & Reference & & \\
\hline & Unmarried & 1.2 & $1.035-1.39$ & $0.016^{*}$ & 1.298 & $1.117-1.508$ & $0.001^{* *}$ \\
\hline \multirow{4}{*}{ Race } & & & & $0.003^{* *}$ & & & 0.11 \\
\hline & White & Reference & & & & & \\
\hline & Black & 0.757 & $0.591-0.971$ & $0.028^{*}$ & & & 0.47 \\
\hline & Other & 0.712 & $0.559-0.906$ & $0.006^{* *}$ & & & 0.062 \\
\hline \multirow{3}{*}{ Sex } & & & & 0.516 & & & \\
\hline & Male & Reference & & & & & NI \\
\hline & Female & & & 0.516 & & & \\
\hline \multirow{5}{*}{ Grade } & & & & $<0.001^{* * *}$ & & & 0.06 \\
\hline & I & Reference & & & Reference & & \\
\hline & II & 1.385 & $1.071-1.79$ & $0.013^{*}$ & 1.234 & $0.952-1.600$ & 0.112 \\
\hline & III & 1.947 & $1.503-2.523$ & $<0.001^{* * *}$ & 1.237 & $0.947-1.615$ & 0.119 \\
\hline & IV & 4.396 & $2.474-7.81$ & $<0.001^{* * *}$ & 2.295 & $1.282-4.109$ & $0.005^{* *}$ \\
\hline \multirow{5}{*}{ T stage } & & & & $<0.001^{* * *}$ & & & $<0.001^{* * *}$ \\
\hline & $\mathrm{T} 1$ & Reference & & & Reference & & \\
\hline & $\mathrm{T} 2$ & 1.565 & $1.112-2.202$ & $0.01^{*}$ & 1.229 & $0.868-1.741$ & 0.245 \\
\hline & $\mathrm{T} 3$ & 3.639 & $2.59-5.114$ & $<0.001^{* * *}$ & 2.424 & $1.699-3.459$ & $<0.001^{* * *}$ \\
\hline & $\mathrm{T} 4$ & 6.902 & $4.287-11.112$ & $<0.001^{* * *}$ & 3.715 & $2.262-6.101$ & $<0.001^{* * *}$ \\
\hline \multirow{4}{*}{ LODDS } & & & & $<0.001^{* * *}$ & & & $<0.001^{* * *}$ \\
\hline & LODDS1 & Reference & & & Reference & & \\
\hline & LODDS2 & 1.525 & $1.262-1.842$ & $<0.001^{* * *}$ & 1.316 & $1.087-1.593$ & $0.005^{* *}$ \\
\hline & LODDS3 & 3.228 & $2.659-3.919$ & $<0.001^{* * *}$ & 2.515 & $2.053-3.080$ & $<0.001^{* * *}$ \\
\hline \multirow{3}{*}{ M stage } & & & & $<0.001^{* * *}$ & & & \\
\hline & M0 & Reference & & & Reference & & \\
\hline & M1 & 3.04 & $2.531-3.651$ & $<0.001^{* * *}$ & 2.067 & $1.704-2.508$ & $<0.001^{* * *}$ \\
\hline \multirow{4}{*}{ LN surgery scope } & & & & $0.003^{* *}$ & & & \\
\hline & None & Reference & & & & & NI \\
\hline & $1 \sim 3$ & 1.462 & $0.825-2.593$ & 0.193 & & & \\
\hline & $\geq 4$ & 1.12 & $0.625-2.005$ & 0.704 & & & \\
\hline \multirow{3}{*}{ Radiation } & & & & 0.289 & & & \\
\hline & Yes & Reference & & & & & NI \\
\hline & No/unknown & 1.217 & $0.991-1.493$ & 0.289 & & & \\
\hline \multirow{3}{*}{ Chemotherapy } & & & & 0.512 & & & \\
\hline & Yes & Reference & & & & & NI \\
\hline & No/unknown & 1.084 & $0.925-1.271$ & 0.512 & & & \\
\hline \multirow{4}{*}{ Tumor size $(\mathrm{cm})$} & & & & $<0.001^{* * *}$ & & & $0.003^{* *}$ \\
\hline & $<1.9$ & Reference & & & Reference & & \\
\hline & $1.9 \sim 4.8$ & 1.605 & $1.31-1.965$ & $<0.001^{* * *}$ & 1.176 & $0.952-1.453$ & 0.132 \\
\hline & $\geq 4.8$ & 2.611 & $2.084-3.271$ & $<0.001^{* * *}$ & 1.503 & $1.179-1.916$ & $0.001^{* *}$ \\
\hline
\end{tabular}

AJCC: American Joint Committee on Cancer; LODDS: log of positive lymph nodes. Level of significance: ${ }^{*} P<0.05 ;{ }^{* *} P<0.01 ;{ }^{* * *} P<0.001$. 


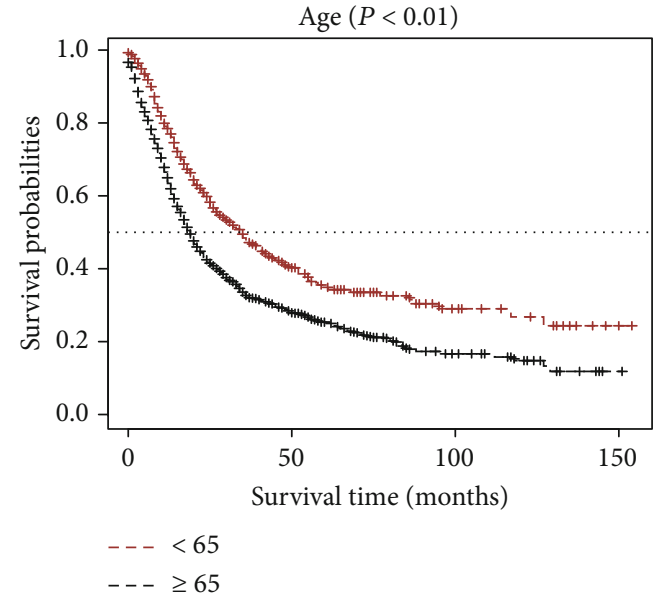

(a)

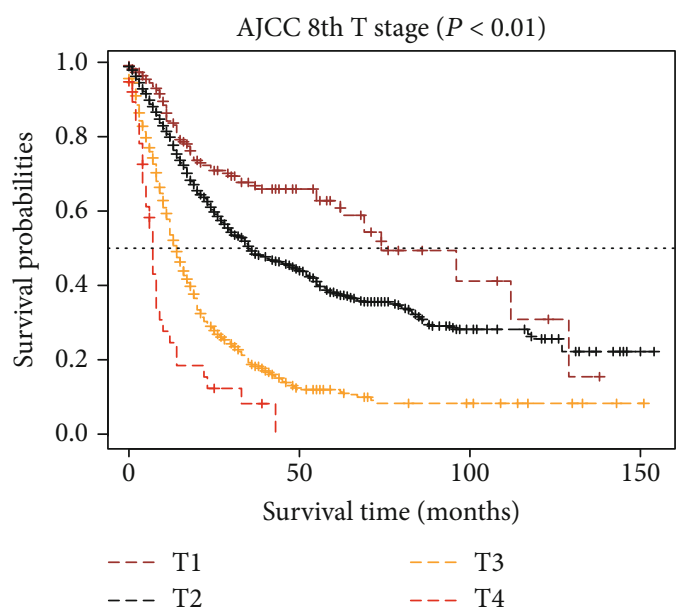

(c)

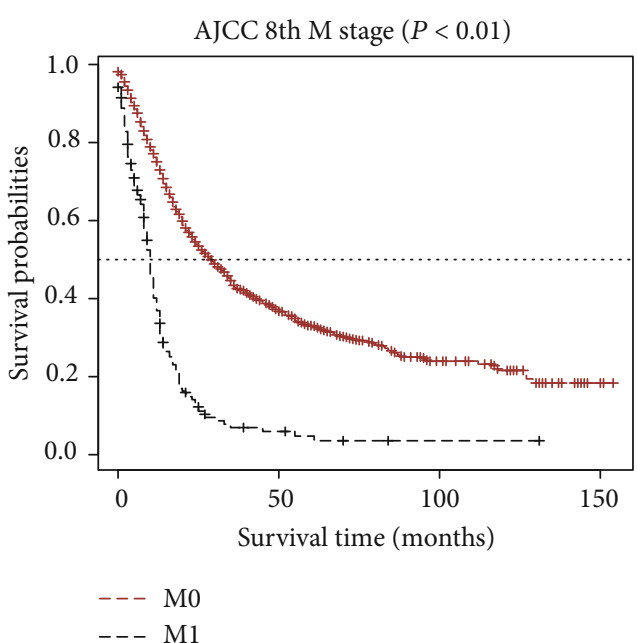

(e)

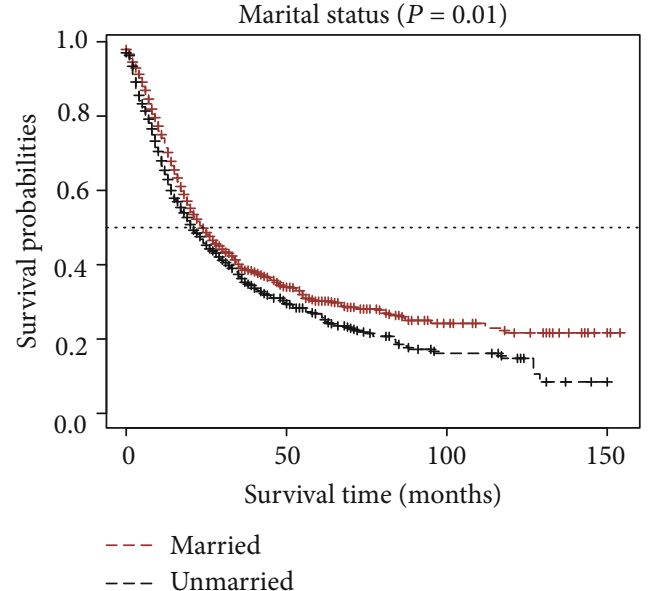

(b)

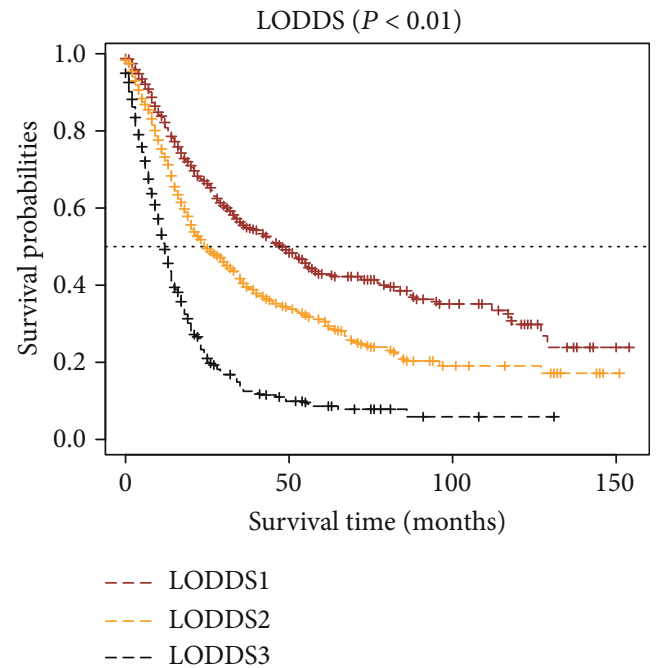

(d)

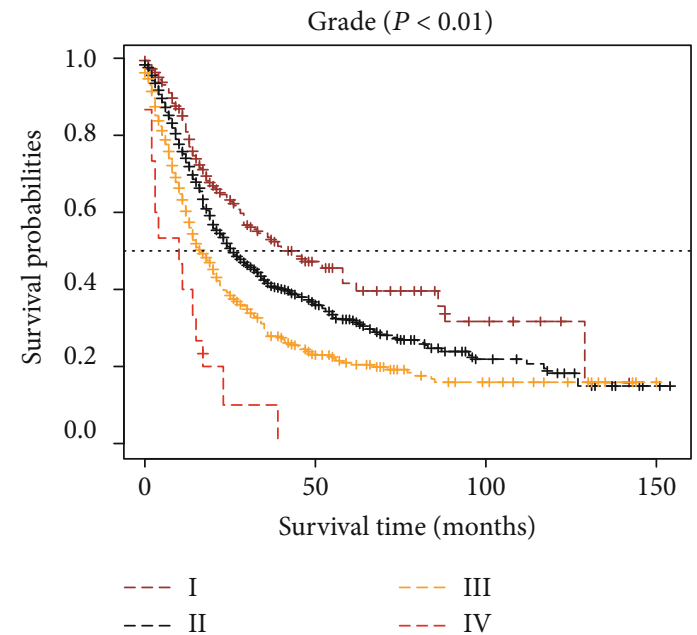

(f)

Figure 2: Continued. 


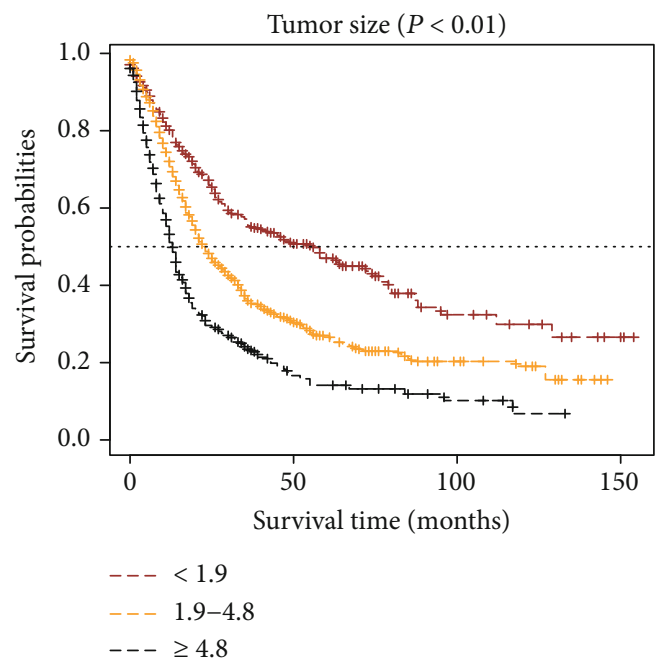

(g)

Figure 2: KM OS curves stratified by independent prognostic factors: (a) age, (b) marital status, (c) AJCC 8th T stage, (d) LODDS, (e) AJCC 8th M stage, (f) tumor grade, and (g) tumor size. AJCC: American Joint Committee on Cancer; LODDS: log of positive lymph nodes.

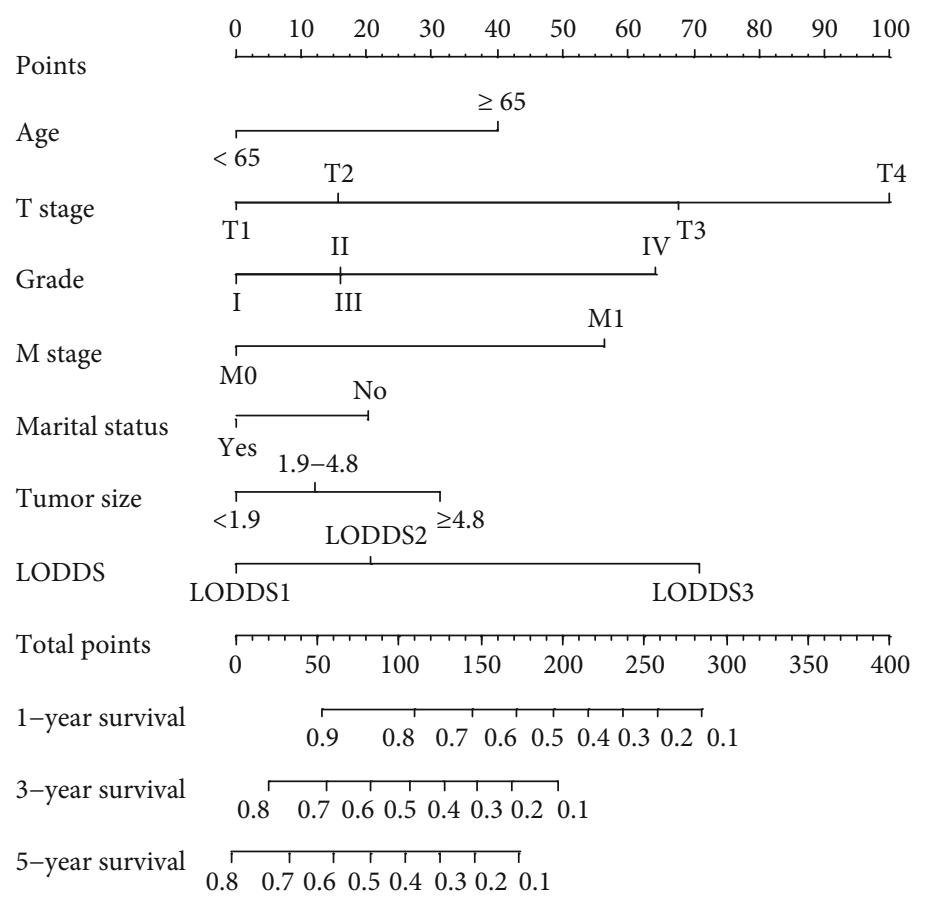

Figure 3: Nomogram to predict 1-, 3-, and 5-year overall survivals in patients who underwent gallbladder adenocarcinoma resection. LODDS: $\log$ of positive lymph nodes.

nodes is closely related to the prognosis of patients with malignant tumors, such as GBC $[24,26-29,32]$. The AJCC recommends the removal of at least 12 lymph nodes to be sufficient for accurate tumor staging [10]. The reason is as we mentioned before. X-tile was developed by Yale University and has been proven to provide an accurate cut-off value for index stratification [33]. It has been widely applied in the evaluation index stratification of different diseases, and we used it to stratify tumor size $[14,34]$. From our results, a larger tumor size indicated a worse prognosis in different tumors $[9,30]$. Compared with tumors smaller than $1.9 \mathrm{~cm}$, tumors larger than $4.8 \mathrm{~cm}$ differed by nearly $30 \%$ in the five-year survival rate in our survival curve.

In our research, we set up a modeling cohort and an internal validation cohort according to previous studies [13, 14]. The C-index in our training cohort was 0.735 (95\% CI, 0.716 to 0.754 ), which denoted medium prediction accuracy (0.71-0.90). It demonstrated better discrimination and ability 


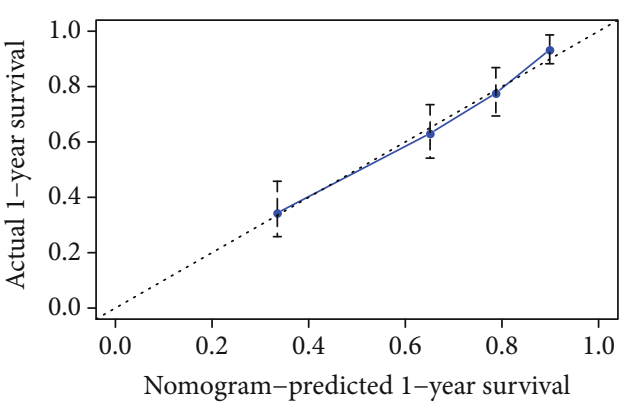

(a)

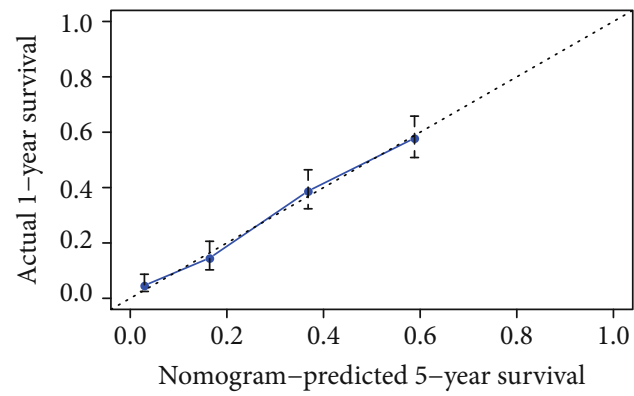

(c)

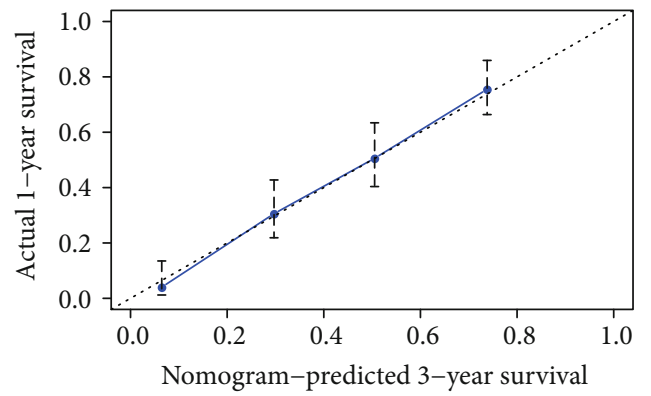

(e)

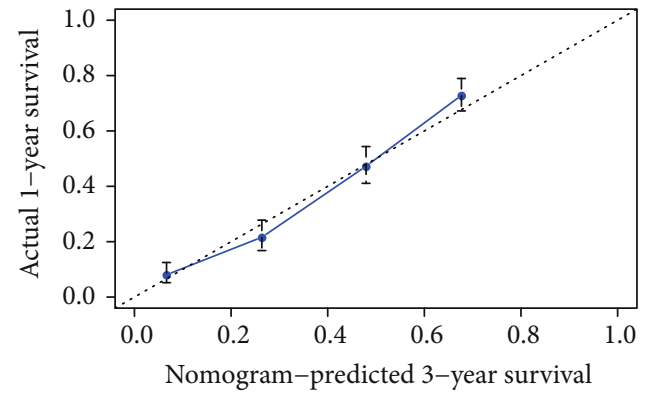

(b)

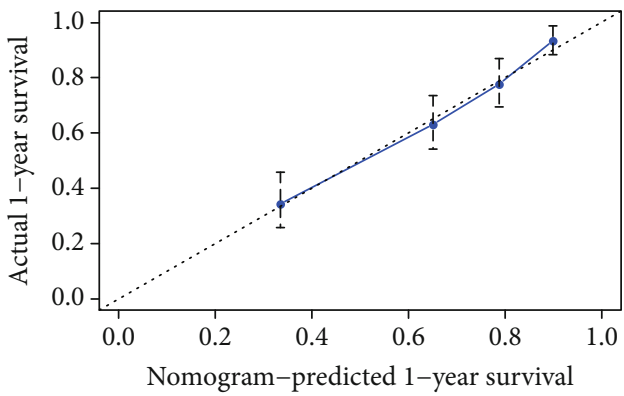

(d)

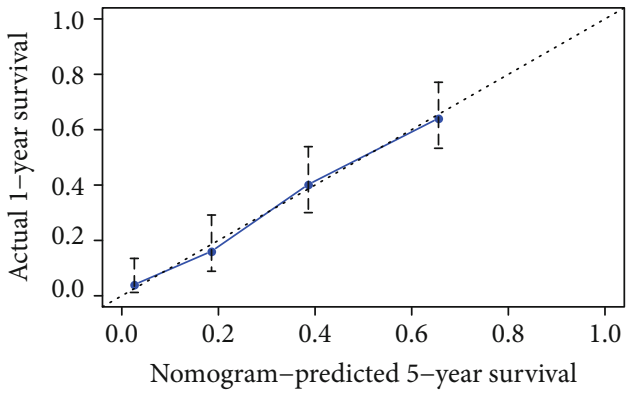

(f)

FIGURE 4: 1-, 3-, and 5-year OS forecasted nomogram calibration graphs for the training cohort (a-c) and internal validation cohort (d-f). The $x$-axis represents the OS probability predicted by the nomogram; the $y$-axis represents the actual survival rate. Diagonal lines represent ideal reference column diagrams. Striped dots represent the probability of the nomogram prediction and the $95 \%$ confidence interval.

to provide patients with personalized predictions. The AIC of our nomogram was 8658 . We found that our nomogram based on LODDS had a more accurate evaluation effect than the $8^{\text {th }}$ edition of the AJCC TNM staging system (C-index: 0.693, 95\% CI: 0.674-0.712; AIC: 8763). Through calibration curve and ROC curve verification, our model showed the expected consistency for predicting 1-, 3-, and 5-year overall survival. These results mean that our nomogram could accurately judge the prognosis of patients with gallbladder adenocarcinoma resection over 45 years old. Utilizing the survival prognosis nomogram we obtained, gallbladder adenocarcinoma patients whose tumor sizes were both $3 \mathrm{~cm}$ were taken as examples (Table 3): A 55-year-old married patient with AJCC TNM stage T2, M0, LODDS2, and pathological grade II scored 78 points, and the 1-, 3-, and 5-year OS rates were $87 \%, 63 \%$, and $53 \%$; the other patient was 75 years old and unmarried with AJCC TNM stage T2, M0, LODDS2, and pathological grade IV and scored 186 points; the 1-, 3-, and 5 -year OS rates were $55 \%, 15 \%$, and $0 \%$. Through these results, we can see that, unlike the traditional AJCC TNM stage, even if two patients have the same TNM stage, we can obtain different prognosis predictions through our nomogram. Using our nomogram, different evaluation indicators can be accurately calculated by scores, and the score of each indicator can be further added to the total score. Through the total score, we can accurately predict the 1-, 3-, and 5-year OS rates of patients. Our nomogram provides a more accurate prognostic evaluation for patients with gallbladder adenocarcinoma. We are confident that our nomogram can help clinicians to make a good assessment of the prognosis of patients undergoing gallbladder adenocarcinoma resection who are older than 45 years.

Our research has some strengths. First, our nomogram was based on the SEER database, which collected clinical data from $28 \%$ of the US population. This means that our model is supported by a large amount of data. Second, compared with previous studies, our model targeted patients with gallbladder adenocarcinoma older than 45 years old, who account for the majority of GBC patients. Finally, calibration curve and ROC curve verification found that our nomogram 


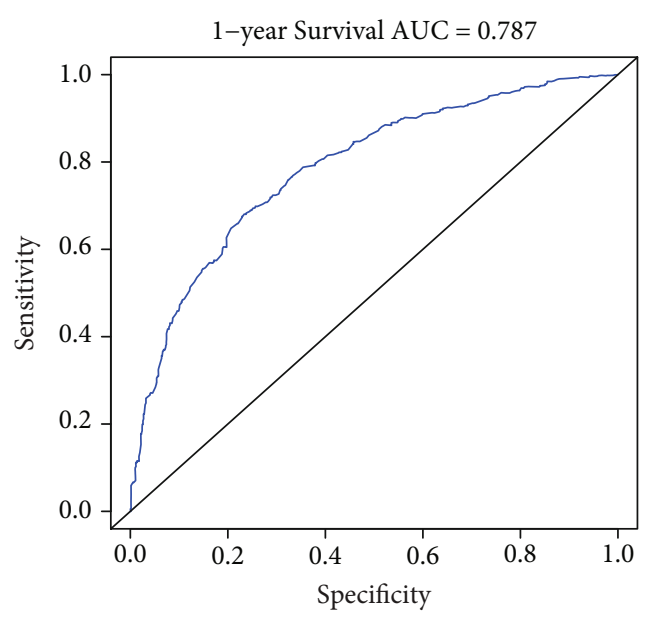

(a)

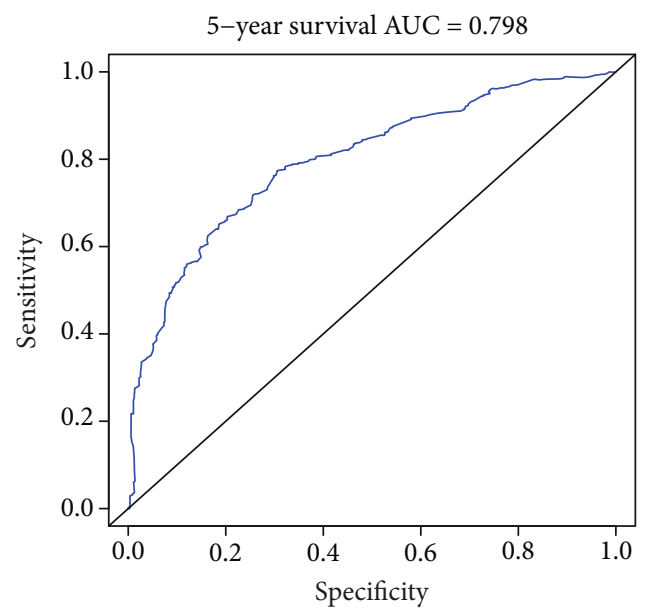

(c)

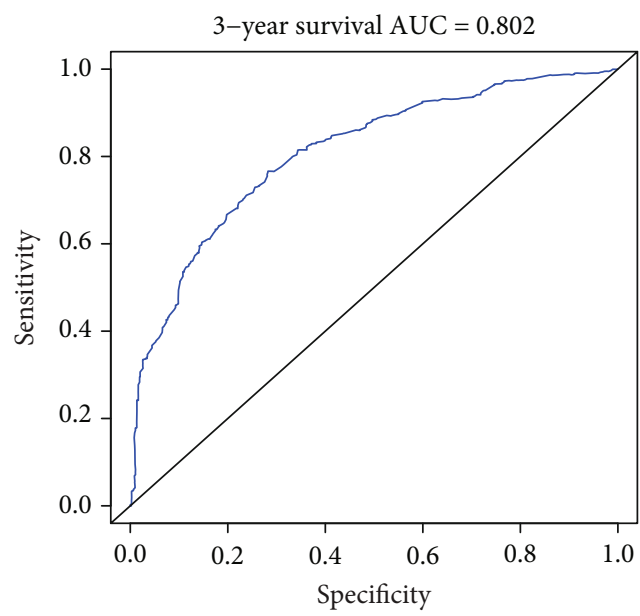

(e)

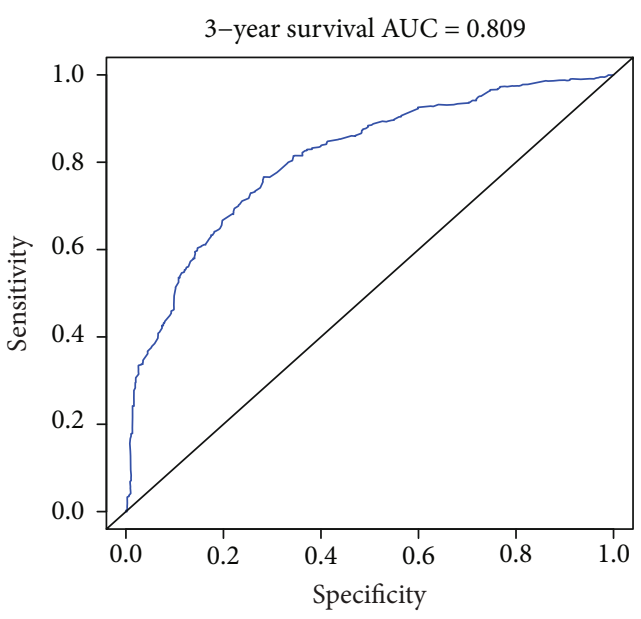

(b)

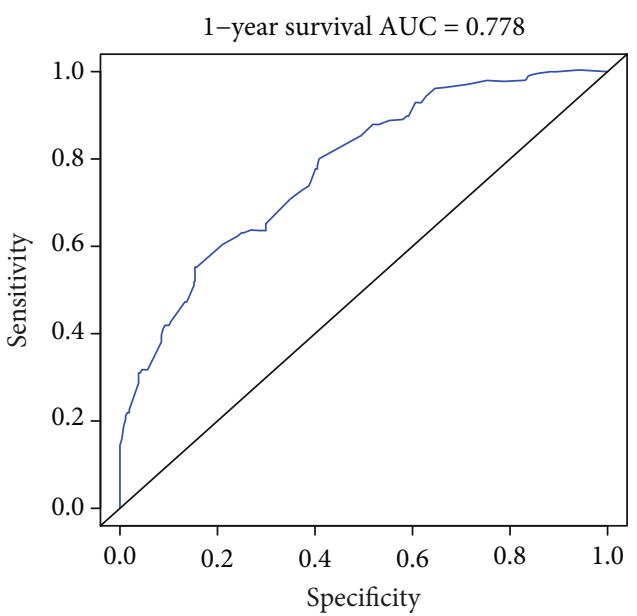

(d)

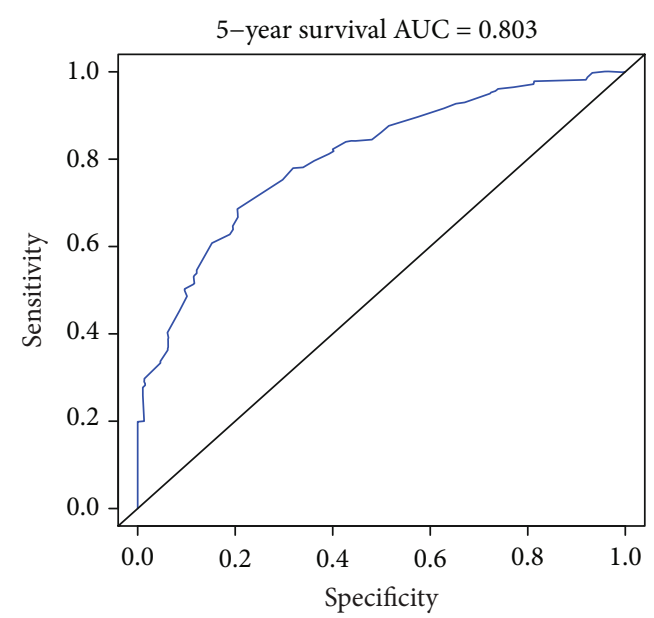

(f)

FIgURE 5: AUC value of the ROC predicting approximately 1-, 3-, and 5-year OS forecasted nomograms for the training cohort (a-b) and internal validation cohort $(\mathrm{d}-\mathrm{f})$.

showed good consistency in the modeling cohort and verification cohort, and the C-index and AIC were better than that of the AJCC $8^{\text {th }}$ TNM stage. This means that our nomogram has a good prognostic effect.
There are also some limitations. First, this was a retrospective study based on the SEER database, so selective bias was inevitable. Second, the establishment of our nomogram has only passed the internal verification of the dataset and 
TABLE 3: Comparison of 1-, 3-, and 5-year OS of two gallbladder adenocarcinoma patients with a tumor size of $3 \mathrm{~cm}$ according to the nomogram.

\begin{tabular}{|c|c|c|c|c|c|c|}
\hline \multirow{2}{*}{ Variable } & \multicolumn{3}{|c|}{ Patient 1} & \multicolumn{3}{|c|}{ Patient 2} \\
\hline & Value & Points & 1-, 3-, 5-year OS & Value & Points & 1-, 3-, 5-year OS \\
\hline Age & 55 & 0 & & 75 & 40 & \\
\hline Marital status & Married & 0 & & Unmarried & 18 & \\
\hline Grade & II & 15 & & IV & 65 & \\
\hline AJCC $\mathrm{T}$ stage & $\mathrm{T} 2$ & 14 & & $\mathrm{~T} 2$ & 14 & \\
\hline AJCC $M$ stage & M0 & 0 & & M0 & 0 & \\
\hline LODDS & LODDS2 & 38 & & LODDS2 & 38 & \\
\hline Tumor size $(\mathrm{cm})$ & 3 & 11 & & 3 & 11 & \\
\hline Total points & & 78 & $87 \%, 63 \%, 53 \%$ & & 186 & $55 \%, 15 \%, 0 \%$ \\
\hline
\end{tabular}

AJCC: American Joint Committee on Cancer; LODDS: log of positive lymph nodes.

lacks external data verification as support. Third, the SEER database cannot obtain the basic indications of patients and the occurrence of complications; thus, the impact of these factors on prognosis could not be evaluated. Future studies need to build a more comprehensive nomogram to provide perfect predictions for patient prognosis.

\section{Conclusions}

Age, marital status, grade, tumor size, AJCC $8^{\text {th }}$ edition T stage, AJCC $8^{\text {th }}$ edition M stage, and LODDS were independent prognostic factors for patients over 45 years old with gallbladder adenocarcinoma. Using these factors, we established a new nomogram as an accurate and convenient method for the assessment of patient prognosis. Our nomogram has a more accurate prognostic evaluation ability than the $8^{\text {th }}$ edition of the AJCC TNM stage.

\section{Abbreviations}

GBC: Gallbladder cancer

LODDS: Log of positive lymph nodes

AJCC: American Joint Committee on Cancer

OS: Overall survival

SEER: Surveillance, Epidemiology, and End Results

C-index: Concordance index

AUC: Area under the curve

ROC: Receiver operating characteristic.

\section{Data Availability}

The data used to support the findings of this study are included within the article.

\section{Conflicts of Interest}

The authors have no conflicts of interest to declare.

\section{Authors' Contributions}

LS contributed to the study design and final manuscript approval. PL and LS performed the statistical analyses and prepared the relevant tables and graphs. PL drafted the man- uscript, and LS revised it. Each of our authors had carefully read and approved the eventual manuscript for publication.

\section{Acknowledgments}

We appreciate the SEER program for providing free access to the database. This study was supported by a grant from the Research Project of Zhongshan Hospital, Fudan University (2016ZSFZ43).

\section{Supplementary Materials}

Figure S1: X-tile plots identifying the cut-off of Tumor Size (A, B, C), LODDS (D, E, F). Abbreviations: 16 LODDS: Log odds of positive lymph nodes. Table S1: process of data screening in the SEER database. (Supplementary Materials)

\section{References}

[1] S. Okubo, S. Mitsunaga, Y. Kato et al., "The prognostic impact of differentiation at the invasive front of biliary tract cancer," Journal of Surgical Oncology, vol. 117, no. 6, pp. 1278-1287, 2018.

[2] I. Baiu and B. Visser, "Gallbladder cancer," JAMA, vol. 320, no. 12, p. 1294, 2018.

[3] S. Sheth, A. Bedford, and S. Chopra, "Primary gallbladder cancer: recognition of risk factors and the role of prophylactic cholecystectomy," The American Journal of Gastroenterology, vol. 95, no. 6, pp. 1402-1410, 2000.

[4] T. O. Goetze, "Gallbladder carcinoma: prognostic factors and therapeutic options," World Journal of Gastroenterology, vol. 21, no. 43, pp. 12211-12217, 2015.

[5] A. Sharma, K. L. Sharma, A. Gupta, A. Yadav, and A. Kumar, "Gallbladder cancer epidemiology, pathogenesis and molecular genetics: recent update," World Journal of Gastroenterology, vol. 23, no. 22, pp. 3978-3998, 2017.

[6] T. Yifan, L. Zheyong, C. Miaoqin, S. Liang, and C. Xiujun, "A predictive model for survival of gallbladder adenocarcinoma," Surgical Oncology, vol. 27, no. 3, pp. 365-372, 2018.

[7] Y. F. Wang, "Combined detection tumor markers for diagnosis and prognosis of gallbladder cancer," World Journal of Gastroenterology, vol. 20, no. 14, pp. 4085-4092, 2014.

[8] B. Gu, L. Qian, H. Yu et al., "Concurrent chemoradiotherapy in curatively resected gallbladder carcinoma: a propensity 
score-matched analysis," International Journal of Radiation Oncology • Biology • Physics, vol. 100, no. 1, pp. 138-145, 2018.

[9] W. Zhang, H. J. Hong, and Y. L. Chen, "Establishment of a gallbladder cancer-specific survival model to predict prognosis in non-metastatic gallbladder cancer patients after surgical resection," Digestive Diseases and Sciences, vol. 63, no. 9, pp. 2251-2258, 2018.

[10] Y. S. Chun, T. M. Pawlik, and J. N. Vauthey, "8th Edition of the AJCC Cancer Staging Manual: pancreas and hepatobiliary cancers," Annals of Surgical Oncology, vol. 25, no. 4, pp. 845-847, 2018.

[11] S. Conci, A. Ruzzenente, M. Sandri et al., "What is the most accurate lymph node staging method for perihilar cholangiocarcinoma? Comparison of UICC/AJCC $\mathrm{pN}$ stage, number of metastatic lymph nodes, lymph node ratio, and log odds of metastatic lymph nodes," European Journal of Surgical Oncology, vol. 43, no. 4, pp. 743-750, 2017.

[12] L. Han, S. Mo, W. Xiang et al., "Comparison of four lymph node staging systems for predicting prognosis for stage IV rectum cancer," Annals of Translational Medicine, vol. 8, no. 4, p. 111, 2020.

[13] Y. Wang, J. Li, Y. Xia et al., "Prognostic nomogram for intrahepatic cholangiocarcinoma after partial hepatectomy," Journal of Clinical Oncology, vol. 31, no. 9, pp. 1188-1195, 2013.

[14] J. Li and L. Liu, "Overall survival in patients over 40 years old with surgically resected pancreatic carcinoma: a SEER-based nomogram analysis," BMC Cancer, vol. 19, no. 1, p. 726, 2019.

[15] S. J. Wang, C. D. Fuller, J. S. Kim, D. F. Sittig, C. R. Thomas Jr., and P. M. Ravdin, "Prediction model for estimating the survival benefit of adjuvant radiotherapy for gallbladder cancer," Journal of Clinical Oncology, vol. 26, no. 13, pp. 2112-2117, 2008.

[16] S. J. Wang, A. Lemieux, J. Kalpathy-Cramer et al., "Nomogram for predicting the benefit of adjuvant chemoradiotherapy for resected gallbladder cancer," Journal of Clinical Oncology, vol. 29, no. 35, pp. 4627-4632, 2011.

[17] X. Li, Y. Liu, Y. Wang et al., "The influence of marital status on survival of gallbladder cancer patients: a population-based study," Scientific Reports, vol. 7, no. 1, p. 5322, 2017.

[18] M. Akce, K. Zakka, M. K. Penley et al., "Clinicopathological features and survival outcomes of rare histologic variants of gallbladder cancer," Journal of Surgical Oncology, vol. 121, no. 2, pp. 294-302, 2019.

[19] W. Song, D. L. Miao, and L. Chen, "Survival rates are higher in married patients with biliary tract cancer: a population-based study," Oncotarget, vol. 9, no. 10, pp. 9531-9539, 2018.

[20] G. Goldzweig, E. Andritsch, A. Hubert et al., "Psychological distress among male patients and male spouses: what do oncologists need to know?," Annals of Oncology, vol. 21, no. 4, pp. 877-883, 2010.

[21] M. Baine, F. Sahak, C. Lin, S. Chakraborty, E. Lyden, and S. K. Batra, "Marital status and survival in pancreatic cancer patients: a SEER based analysis," PLoS One, vol. 6, no. 6, article e21052, 2011.

[22] C. Chen, Z. Geng, H. Shen et al., "Long-term outcomes and prognostic factors in advanced gallbladder cancer: focus on the advanced T stage," PLoS One, vol. 11, no. 11, article e0166361, 2016.

[23] Z. Q. Cai, P. Guo, S. B. Si, Z. M. Geng, C. Chen, and L. L. Cong, "Analysis of prognostic factors for survival after surgery for gallbladder cancer based on a Bayesian network," Scientific Reports, vol. 7, no. 1, p. 293, 2017.

[24] E. Sasaki, M. Nagino, T. Ebata et al., "Immunohistochemically demonstrated lymph node micrometastasis and prognosis in patients with gallbladder carcinoma," Annals of Surgery, vol. 244, no. 1, pp. 99-105, 2006.

[25] T. B. Tran and N. N. Nissen, "Surgery for gallbladder cancer in the US: a need for greater lymph node clearance," Journal of gastrointestinal oncology, vol. 6, no. 5, pp. 452-458, 2015.

[26] E. Vo, S. A. Curley, C. Y. Chai, N. N. Massarweh, and H. S. Tran Cao, "National failure of surgical staging for T1b gallbladder cancer," Annals of Surgical Oncology, vol. 26, no. 2, pp. 604-610, 2019.

[27] K. Toge, J. Sakata, Y. Hirose et al., "Lymphatic spread of T2 gallbladder carcinoma: regional lymphadenectomy is required independent of tumor location," European Journal of Surgical Oncology, vol. 45, no. 8, pp. 1446-1452, 2019.

[28] S. H. Kim, J. U. Chong, J. H. Lim et al., "Optimal assessment of lymph node status in gallbladder cancer," European Journal of Surgical Oncology, vol. 42, no. 2, pp. 205-210, 2016.

[29] J. Y. Lin, D. S. Bai, B. H. Zhou et al., "Positive relationship between number of negative lymph nodes and duration of gallbladder cancer cause-specific survival after surgery," Cancer Management and Research, vol. Volume 10, pp. 6961-6969, 2018.

[30] Z. Xiao, Z. Shi, L. Hu et al., "A new nomogram from the SEER database for predicting the prognosis of gallbladder cancer patients after surgery," Annals of Translational Medicine, vol. 7, no. 23, p. 738, 2019.

[31] S. Occhionorelli, D. Andreotti, P. Vallese et al., "Evaluation on prognostic efficacy of lymph nodes ratio (LNR) and log odds of positive lymph nodes (LODDS) in complicated colon cancer: the first study in emergency surgery," World Journal of Surgical Oncology, vol. 16, no. 1, p. 186, 2018.

[32] R. K. Chaudhary, R. Higuchi, T. Yazawa et al., "Surgery in node-positive gallbladder cancer: the implication of an involved superior retro-pancreatic lymph node," Surgery, vol. 165, no. 3, pp. 541-547, 2019.

[33] R. L. Camp, M. Dolled-Filhart, and D. L. Rimm, "X-tile: a new bio-informatics tool for biomarker assessment and outcomebased cut-point optimization," Clinical Cancer Research, vol. 10, no. 21, pp. 7252-7259, 2004.

[34] Y. Li, L. Liang, W. Dai et al., "Prognostic impact of programed cell death-1 (PD-1) and PD-ligand 1 (PD-L1) expression in cancer cells and tumor infiltrating lymphocytes in colorectal cancer," Molecular Cancer, vol. 15, no. 1, p. 55, 2016. 